\title{
Rapid Rapamycin-Only Induced Osteogenic Differentiation of Blood-Derived Stem Cells and Their Adhesion to Natural and Artificial Scaffolds
}

\author{
Carpentieri Arianna, ${ }_{1}^{1}$ Cozzoli Eliana, ${ }^{1}$ Acri Flavio, ${ }^{2}$ Ranalli Marco, ${ }^{1}$ Diedenhofen Giacomo, ${ }^{1}$ \\ Scimeca Manuel, ${ }^{1,3,4}$ Bonanno Elena, ${ }^{1}$ and Gambacurta Alessandra ${ }^{1,5}$ \\ ${ }^{1}$ Biochemistry Laboratory, Department of Experimental Medicine and Surgery, University of Rome "Tor Vergata", 00133 Rome, Italy \\ ${ }^{2}$ Baxter Healthcare Ltd, Caxton Way, Thetford, UK \\ ${ }^{3}$ Department of Biomedicine and Prevention, University of Rome "Tor Vergata", 00133 Rome, Italy \\ ${ }^{4}$ OrchideaLab S.r.l., Via del Grecale 6, Morlupo, Rome, Italy \\ ${ }^{5}$ NAST Centre for Nanoscience, University of Rome "Tor Vergata", 00133 Rome, Italy
}

Correspondence should be addressed to Carpentieri Arianna; arianna.carpentieri@libero.it and

Gambacurta Alessandra; gambacur@uniroma2.it

Received 3 January 2017; Accepted 11 June 2017; Published 26 July 2017

Academic Editor: Boon C. Heng

Copyright (c) 2017 Carpentieri Arianna et al. This is an open access article distributed under the Creative Commons Attribution License, which permits unrestricted use, distribution, and reproduction in any medium, provided the original work is properly cited.

\begin{abstract}
Stem cells are a centerpiece of regenerative medicine research, and the recent development of adult stem cell-based therapy systems has vigorously expanded the scope and depth of this scientific field. The regeneration of damaged and/or degraded bone tissue in orthopedic, dental, or maxillofacial surgery is one of the main areas where stem cells and their regenerative potential could be used successfully, requiring tissue engineering solutions incorporating an ideal stem cell type paired with the correct mechanical support. Our contribution to this ongoing research provides a new model of in vitro osteogenic differentiation using blood-derived stem cells (BDSCs) and rapamycin, visibly expressing typical osteogenic markers within ten days of treatment. In depth imaging studies allowed us to observe the adhesion, proliferation, and differentiation of BDSCs to both titanium and bone scaffolds. We demonstrate that BDSCs can differentiate towards the osteogenic lineage rapidly, while readily adhering to the scaffolds we exposed them to. Our results show that our model can be a valid tool to study the molecular mechanisms of osteogenesis while tailoring tissue engineering solutions to these new insights.
\end{abstract}

\section{Introduction}

Regenerative medicine is an important and relevant scientific research field whose focus on developing cell-based therapeutic approaches to regenerate damaged tissues, or even replace whole organs, has attracted scientists from around the world [1]. One of the main areas where stem cells and their regenerative potential could be used successfully is orthopedic, dental, and maxillofacial surgery [2, 3]. Problems such as the treatment of fractures involving extensive bone damage or a particularly sensitive localization are a complex challenge for surgeons, and there are some pathological conditions that are treatable only insufficiently with conventional implants and surgical procedures [4-6].

The solution can be found in tissue engineering, where cells are combined with a three-dimensional matrix to compose a tissue-like construct to substitute lost tissues, or even whole organs [1]. Adult stem cells have shown remarkable plasticity, and they have been extensively studied for their potential applications in tissue engineering [6, 7].

Today, there are many sources from which it is possible to draw adult stem cells, such as bone marrow, adipose tissue, periosteum, human umbilical cord, and mesenchymal tissue $[8-11]$. 
Presently, mesenchymal stem cells seem to be the most promising candidates for bone regeneration due to their excellent osteogenic differentiation capacity, but they are not without drawbacks as these cells are relatively difficult to expand in vitro, and they lose their regenerative potential during in vitro passages $[12,13]$. Another factor that enormously restricts the use of stem cells as a routine therapeutic tool is that we have little knowledge about the specific pathways they use during differentiation, bone healing, and revascularization [6].

A further prominent aspect in the study of osteogenic differentiation from stem cells is the importance of physical and/or mechanical supports. The role of the scaffold is to support cell growth and differentiation and to control interactions of cells within the biological milieu, while maintaining the mechanical and physical properties required for the selected application [14]. Although implants are successful, it takes about 4-6 months for the healing and integration of implants within the existing bone to occur [14]. Thus, the real challenge in bone regeneration and repair lies in accelerating healing and reducing the recuperation time, since the lack of cell adhesion to substrates commonly used in orthopedic and dental implants remains the leading cause of slower integration and subsequent recovery time $[3,14]$.

The ideal combination of both the regenerative properties of stem cells and the mechanical support provided by implants and scaffolds would require a simple stem cell production method paired with good adherence to the scaffold [15]. Any simplification of the production process makes the eventual procedure more time- and cost-effective, while good adhesion ensures a greater implant success rate. Finding the right combination between stem cell type and threedimensional support/scaffold remains one of the main challenges of regenerative medicine and is particularly relevant to the field of bone tissue regeneration $[16,17]$. This "winning couple" should be suited for both therapeutic and research purposes, in order to elucidate the mechanisms involved in tissue regeneration while providing new tools to the clinician.

However, it is clear that much more research has to be done before we can reach such an ideal stage, as regenerative therapies for bone reconstitution require a deeper understanding of both the biological and the mechanical conditions involved. As with all research endeavors, the creation of new models that may facilitate these studies remains a top priority.

Recently, rapamycin, a selective mTOR (mammalian target of rapamycin) inhibitor, has been shown to induce osteoblastic differentiation in human embryonic stem cells $[18,19]$. This molecule has been shown to act on the PI3K/ $\mathrm{AKT} / \mathrm{mTOR}$ signaling pathways, inducing the upregulation of the early osteogenic markers BMP 2 and Runx2 and modulating the BMP/Smad signaling in human embryonic stem cell (hESC), as well as being important in the regulation of self-renewal and differentiation [20, 21]. Rapamycin is already approved by the FDA for use as a therapeutic agent for humans, making it potentially translatable to orthopedic applications. In fact, it has already shown promise of future applicability in bone regeneration both in vitro and in vivo
[22]. Taking this into consideration, we were thus intrigued by the possibility of further applications of this specific molecule within the setting of a new "in vitro" model of bone differentiation. Having already demonstrated, the plasticity of blood derived-stem cells (BDSCs) [23-26] and knowing their potential applications in bone tissue repair, we tried to develop a reproducible and defined method to investigate their potential for osteogenic/cellular differentiation.

In this paper, we propose an osteogenic differentiation model that makes use of both rapamycin as a single inductor and bone matrix scaffolds. The results obtained show that not only the timing of commitment towards the osteogenic lineage is shortened compared to the literature $[27,28]$ but also it is possible to follow this process through a simple confocal microscopy analysis.

Furthermore, we have also shown the ability of BDSCs to adapt and adhere to different types of scaffolds approved for medical use, particularly in orthopedic and dental surgery.

\section{Materials and Methods}

2.1. Stem Cells Preparation. Human BDSCs were obtained by blood samples as previously described [23-26]. Briefly, the nucleated blood cell fraction was isolated by ammonium chloride incubation (dilution 1:3 in $\mathrm{NH}_{4} \mathrm{Cl} 1 \mathrm{M}$ ), centrifuged at $400 \mathrm{~g}$, and washed several times with phosphate-buffered saline (PBS), pH 7.2 (Oxoid, Hampshire, England, number BR0014G) to remove the majority of erythrocytes. Cells were then resuspended in $5 \mathrm{ml} \mathrm{PBS}$ and incubated for $72 \mathrm{~h}$ at $37^{\circ} \mathrm{C}$ in the presence of $50 \mathrm{nM}$ macrophage colony-stimulating factor (Sigma-Aldrich, St. Louis, MO, number M9170), and $5 \mu \mathrm{M}$ gentamicin sulphate (BioWest, Nuaillé, France, number L0012).

2.2. Osteogenic Differentiation of BDSCs. To promote osteogenic differentiation in vitro, the human BDSCs were plated on a collagenated 24-multiwell plate. The plate coating protocol required a $0.1 \mathrm{mg} / \mathrm{ml}$ type I rat tail collagen, high concentration solution (BD Pharmigen, USA, cat. number 354249), added to the 24-well plates, and incubated for $1 \mathrm{~h}$ at $37^{\circ} \mathrm{C}$. After incubation, the plates were washed three times with PBS and kept at $+4^{\circ} \mathrm{C}$ until use.

We used different procedures to differentiate BDSCs:

(i) Commercial kit: The cells were differentiated using the "Human Mesenchymal Stem Cell Functional Identification Kit" (R\&D systems, number SC006) according to the manufacturer's protocol.

(ii) Rapamycin: The cells were grown in a 24 -well plate for 10 days in a differentiation medium composed by DMEM-F12 supplemented with $15 \mathrm{mM}$ HEPES and $2 \mathrm{mM}$ L-glutamine (Lonza, Swiss, cat. number BE12-719F), 10\% FBS (Lonza, Belgium, cat. number DE14-830F), 1\% penicillin (100 units/ml)/streptomycin $(100 \mathrm{mg} / \mathrm{ml})$ (Lonza, Belgium, cat. number DE17-602E), 1\% nonessential amino acids (Biowest, cat. number X0557-100), and Rapamycin, as osteogenic inductor, at a final concentration of $10 \mathrm{nM}$ (Sigma-Aldrich, cat. number R0395). 
2.2.1. Rapamycin and Scaffolds. The cells were grown for 10 days in the presence of several types of scaffolds in the same differentiation medium as described above.

\subsection{Scaffold Types}

2.3.1. Bone Scaffold. We used the BIO-OSS a spongious bone substitute of bovine origin produced by Geistlich Biomaterials. The granule size was $0.25 \mathrm{~mm}-1 \mathrm{~mm} / 0.5 \mathrm{~g}$ [29].

2.3.2. Titanium Scaffold. We used two titanium commercial products. The first (type 1) from CAMLOG, Alta-Tech Biotechnologies Srl. was obtained by classical endosseous implants and cut into thin slices (suitable for the 24-well plates) with extrathin cutting discs nonpolluting inline () (B.M. DENTALE S.a.s.). The second (type 2) was derived from a titanium grill used in the dental field for guided bone regeneration (TITANGUIDE), produced by Prodent Italia S.r.l. In this case, the original surface of each plate $(20.5 \mathrm{~mm} \times 30 \mathrm{~mm} \times 0.13 \mathrm{~mm})$ possesses numerous microholes $(\varnothing 0.7 \mathrm{~mm})$ and was also cut to obtain rectangles $(5 \times 4 \mathrm{~mm}$ average dimensions) to perform the experiments in the 24-well plates.

Titanium scaffold treatment protocol, to avoid particulates, autoclave sterilization followed by an ultrasonic bath for $5 \mathrm{~min}$ in deionized $\mathrm{H}_{2} \mathrm{O}$, and then for $5 \mathrm{~min}$ in $\mathrm{H}_{2} \mathrm{O}_{2}$ at $120 v / v$, and again for $10 \mathrm{~min}$ in deionized $\mathrm{H}_{2} \mathrm{O}$, was performed, as decribed by Di Silvestro et al. [30]. Furthermore, to avoid alterations of the superficial layer of the scaffolds, an important factor in cell/surface interaction, particular care was taken in performing any and all manipulations of the titanium scaffolds using only plastic or pure titanium instruments.

2.4. Alizarin Red S Staining. The presence of calcium phosphate deposits in BDSCs following differentiation was evaluated by staining with Alizarin Red S (Sigma-Aldrich, Germany, cat. number A5533-25G). The cells were fixed with $4 \%[v / v]$ formaldehyde, stained with $1 \%[v / v]$ Alizarin Red S, and incubated at room temperature in the dark for 20 minutes. The excess of dye was removed with distilled water, and the cells were observed by optical microscopy.

2.5. Immunofluorescence Analyses. The cells were washed twice with PBS and fixed on a glass slide with $4 \%$ formalin (ScyTek Laboratories, Logan, UT) for $30 \mathrm{~min}$ at room temperature (RT), then washed with PBS, and permeabilized/ blocked with $0.3 \%$ Triton X-100, goat serum $5 \%$, and $1 \%$ BSA in PBS for $45 \mathrm{~min}$ at RT. After adding blocking solution, the washed cells were incubated with the primary antibody mouse anti-osteocalcin (1:100 R\&D System 962643 ) overnight at $4^{\circ} \mathrm{C}$. Subsequently, the cells were incubated for 1 hour in the dark at RT with the secondary antibody anti-mouse Alexafluor 488 (1:1000) (Invitrogen, Life Technologies Corporation, NY) and with $1 \mu \mathrm{g} / \mathrm{ml}$ of 40,6-diamidino-2-phenylindole (DAPI, Sigma) to stain cells nuclei.
2.6. Time Course Experiments on Titanium. We performed a time-dependent analysis to evaluate cellular growth and adhesion on both types of titanium. Briefly, seven type 2 titanium scaffolds were placed in seven separate wells, all treated as previously described. Every day for a week, one scaffold was taken and fixed in formalin $4 \%$, washed with PBS after 20 minutes, and prepared for confocal microscopy analysis in order to have a full picture of the cellular growth.

Two types of analyses were performed:

(i) Densitometric analysis: To determine the cells' adhesion to the titanium scaffold, we calculated the increase in average luminosity of DAPI over time executed on the blue fluorescence channel using the Nikon EZ-C1 Viewer software.

To verify that data obtained from the analysis was not simply influenced by an increase of background noise with a correlated increase of average luminosity, we controlled the intensity of luminosity of the same channel along a random direction of the image.

(ii) Coverage analysis: To calculate the percentage area of scaffold covered by cells, an analysis of intensity of luminosity per single pixel was performed using the NIS Elements Viewer provided by Nikon (Copyright (C) 2005 Macrovision Corporation). Based on a control sample, the lowest and highest luminosity values were ignored in order to eliminate both, the background noise, and the peaks with a too high luminosity levels.

All images have been shot at 12 bit, therefore presenting a resolution of $4096\left(2^{12}\right)$ luminosity levels per pixel. On a scale from 0 to 4095, all pixels with an ADC (analog to digital converter) resolution below 156 units and all those with an ADC above 2000 were ignored. All signals within this window have been recorded, yielding a percentage of scaffold surface area covered by cellular growth.

2.7. Protein Extraction and Western Blot Analysis. Cells were homogenized directly into the following buffer: Tris $50 \mathrm{mM}$, $\mathrm{NaCl} 150 \mathrm{mM}$, EDTA $10 \mathrm{mM}$, and Triton-X $1 \%$ and centrifuged at $10,000 \mathrm{~g}$ for $10 \mathrm{~min}$. Protein concentrations were determined by the Bradford assay. Proteins were resolved by $16 \%$ SDS-PAGE, electrotransferred on PVDF membranes (Amersham $^{\mathrm{TM}}$ Hybond $^{\mathrm{TM}}$, GE Healthcare Life Science, cat. number 28906837), and blocked with 5\% $[v / v]$ nonfat dry milk/0.1\% $[v / v]$ TBS-T. The blots were probed with the following primary antibodies: mouse monoclonal antiosteocalcin (1:500) (abcam-ab13420) in 5\% BSA/TBS-T $0.1 \%$ and mouse monoclonal anti-beta actin $(1: 10,000)$ (sigma-aldrich-A5541).

Membranes were then incubated with the appropriate horseradish peroxidase-conjugated donkey anti-mouse secondary antibody (1:5000; Jackson), and the reaction was detected with the Western lighting Plus ECL (Perkin Elmer, Waltham, MA, USA). 
2.8. Scanning Electron Microscopy (SEM) and Energy Dispersive X-Ray (EDX) Microanalysis. Small scaffold supports were fixed in $4 \%(v / v)$ paraformaldehyde and postfixed in $2 \%$ osmium tetroxide. After washing with $0.1 \mathrm{M}$ phosphate buffer, the sample was dehydrated by a series of incubations in $30 \%, 50 \%$, and $70 \%(v / v)$ ethanol. Dehydration was continued by incubation steps in $95 \%(v / v)$ ethanol, absolute ethanol, and acetone. Critical-point drying (Agar Scientific, ElektronTechnology UK Ltd) with supercritical $\mathrm{CO}_{2}$ was then performed to prevent cell deformation. Surfaces of the scaffolds were coated with gold and scanned using SEM LEO 1450VP (Carl Zeiss Meditec, Germany) [31]. EDX microanalysis was performed on exosome using a liquid $\mathrm{N}_{2}$-cooled Si detector with a super-ultrathin Be window. Spectra were collected by a SEM LEO 1450VP scanning electron microscope at acceleration voltage of $5 \mathrm{KeV}$ employing an area scan mode $(640 \times 640 \mu \mathrm{m}$ sampling area), $300 \mathrm{~s}$ acquisition time, and $32-37 \%$ detector dead time. Analysis of acquired spectra was performed under a nonstandard mode using atomic number-absorption-florescence correction (ZAF) methods using Inca Energy software (Oxford Instruments Ltd., High Wycombe, UK; $\mathrm{Si}(\mathrm{Li})$ detector, ATW-atmospheric thin window, resolution $133 \mathrm{eV}$ for $\mathrm{MnK} \alpha$ at 10000 counts).

\subsection{Transmission Electron Microscopy (TEM) and EDX} Microanalysis. Cells were fixed in $4 \%$ paraformaldehyde, postfixed in $2 \%$ osmium tetroxide, and embedded in EPON resin for morphological studies. After washing with $0.1 \mathrm{M}$ phosphate buffer, the sample was dehydrated by a series of incubations in 30\%, 50\%, and 70\% ethanol [32]. Dehydration was continued by incubation steps in $95 \%$ ethanol, absolute ethanol, and hydropropyl methacrylate, and then samples were embedded in Epon (Agar Scientific, Stansted Essex CM24 8GF, United Kingdom). After incubation, cells were cut and stained with heavy metal solutions as described by Reynolds [33]. For the EDX microanalysis, $100 \mathrm{~nm}$ thick unstained ultrathin sections were placed on specific copper grids. The EDX spectra were acquired on matrix vesicles by a Hitachi 7100FA transmission electron microscope and an EDX detector (Thermo Scientific, Waltham, MA, USA) at an acceleration voltage of $75 \mathrm{KeV}$. Spectra were semiquantitatively analyzed by the Noram System Six software (Thermo Scientific, Waltham, MA, USA) using the standardless Cliff-Lorimer k-factor method [34]. The EDX microanalysis system was calibrated using the X-ray microanalysis standard (Micro-Analysis Consultants Ltd, Cambridgeshire, UK).

\section{Results and Discussion}

3.1. BDSCs Successfully Differentiate into Osteogenic Tissue. The BDSCs derived from $72 \mathrm{~h}$ of deprogramming and expansion in PBS, supplemented with MCSF and gentamicin sulphate [23-26], were plated and presented typical morphological features of stemness such as reduced small size, roundish shape, and, in vitro, a disposition to a "string of pearls" morphology (Figure 1(a)). Once we confirmed the achievement of these morphological changes, our
BDSCs were ready to demonstrate their potential for osteogenic differentiation.

We first tested this by utilizing the commercial kit already used in the majority of works on stem cell bone differentiation as our inducer. Our BDSCs were responsive to this treatment and acquired osteoblast-like features within 15 days (data not shown). The commercial differentiation kits are mainly composed by a cocktail of dexamethasone, ascorbic acid, $\beta$-glycerophosphate, and various added excipients. The mechanisms underlying the kit-mediated osteogenic differentiation are only partially known and understood, while the combination of substances used is not approved for human therapy yet. For these reasons, we believe that new differentiation protocols are necessary to put more circumscribed molecular mechanisms into play, with the aim of achieving a higher translational potential to in vivo application.

3.2. Rapamycin Alone Allows for Efficient and Rapid Differentiation. We then attempted this differentiation anew, using only rapamycin $10 \mathrm{nM}$ as an inducer [20-22]. After ten days of rapamycin treatment, we stained the cells with Alizarin Red S to detect the presence of inorganic calcium phosphate deposits and to confirm that osteogenic differentiation occurred (Figure 1(b)). Although the detection of mineralization in confluent monolayers by Alizarin Red S is often considered sufficient evidence to demonstrate a completed osteogenic differentiation [35], we also performed an immunocytochemical, immunofluorescence analysis for detecting osteocalcin, a typical osteogenic marker, as further evidence of the successfully completed process (Figures 1(c) and 1(d) and SD1 available online at https://doi.org/10.1155/2017/2976541). This data suggests that our cells can differentiate within 10 days under the exclusive guide of Rapamycin, making it a good alternative to the commercial kit.

3.3. Bone Scaffolds Improve Rapamycin Protocol Yield. Once we demonstrated, the ability of BDSCs to differentiate into osteoblast-like cells using rapamycin, we performed the same differentiation experiments in the presence of deproteinized and decellularized bovine bone matrix (SD 2). Our aim was to verify whether these cells were able to undergo osteogenic differentiation when in contact with a scaffold and whether the presence of the matrices improved the process. After the full ten-day treatment, we performed an ultrastructural analysis by SEM and TEM (Figures 2(a) and 2(b) and Figures 2(c) and 2(d), resp.), cytochemistry, and Western blot analysis for osteocalcin (SD 2) to verify our hypothesis.

SEM evaluation confirmed that the BDSCs cultured in the presence of rapamycin and bone matrix scaffold underwent morphological changes into osteoblast-like phenotypes, as shown in Figure 2(a), showing extended osteocalcin deposits. In addition, we captured the calcium phosphate-containing exosomes on the surface of osteoblast-like cells (riferimento imagine SEM con lo spettro EDX). 


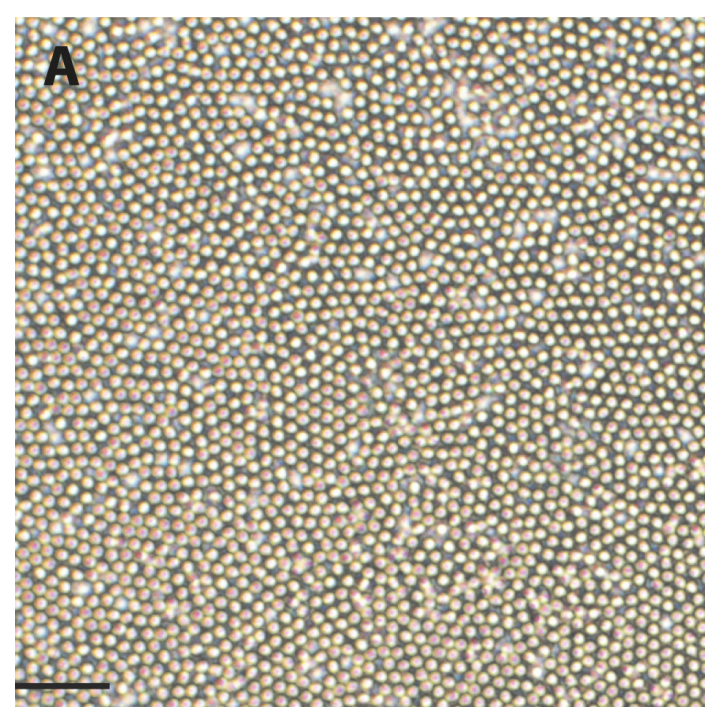

(a)

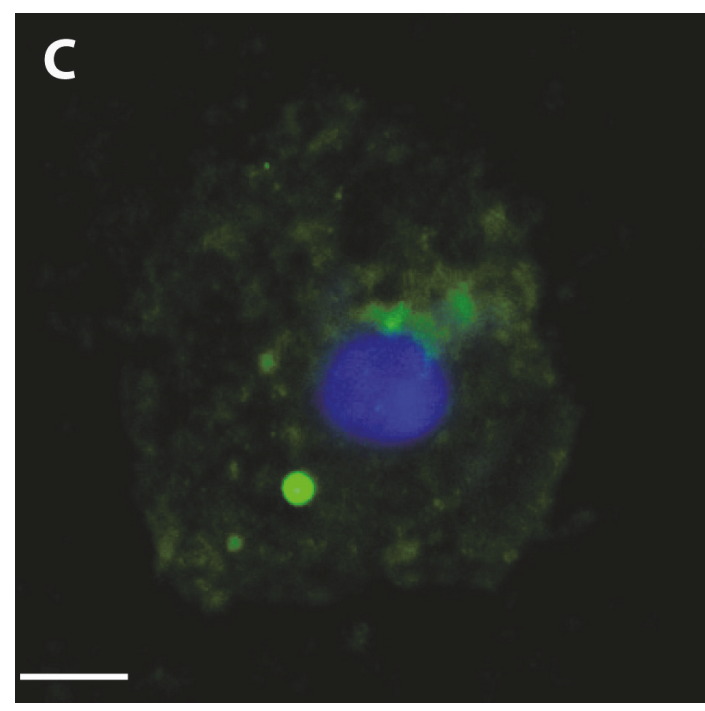

(c)

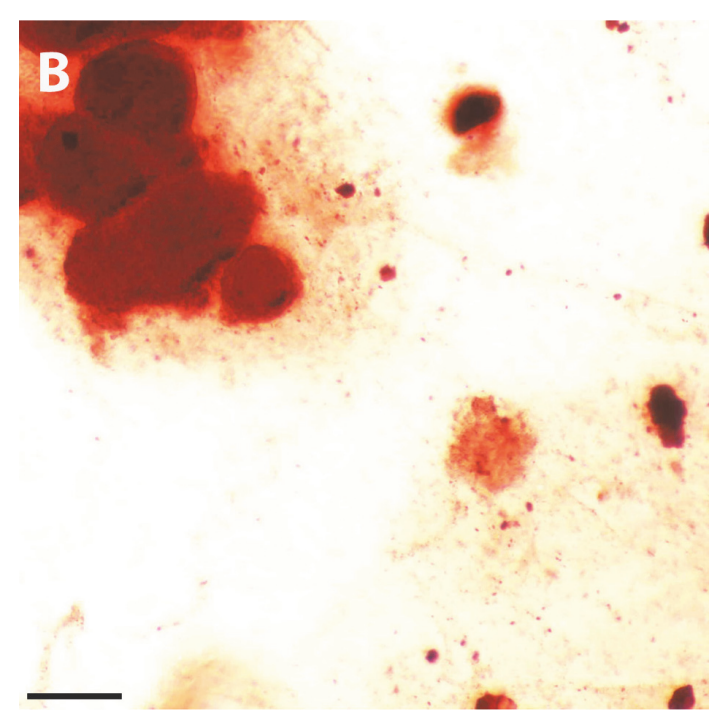

(b)

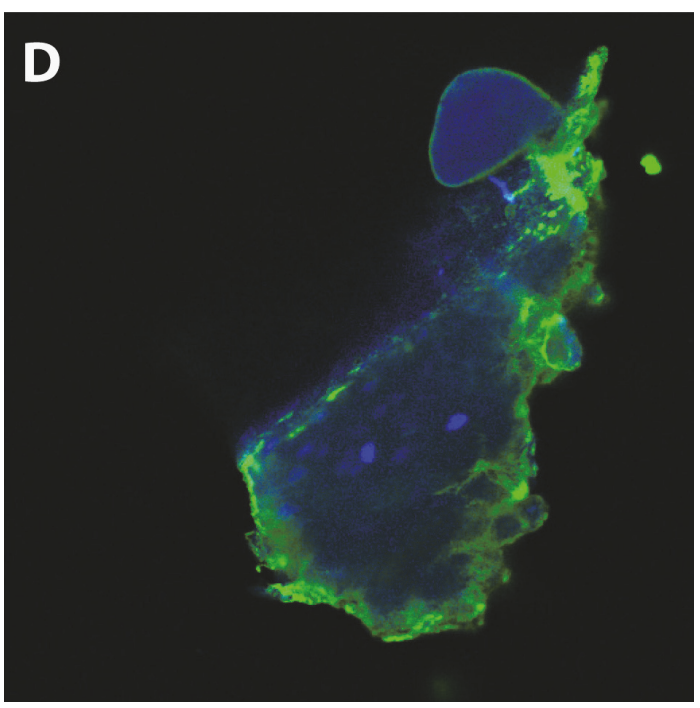

(d)

Figure 1: Blood derived stem cells' (BDSCs) osteogenic differentiation with rapamycin. (a) BDSCs before starting the differentiation protocol. The cells show typical morphological features of stemness such as small size, roundish shape, and, in vitro, a disposition to a "string of pearls" appearance. (b) Alizarin Red S staining on BDSCs after 10 days of osteogenic differentiation, to evaluate inorganic calcium phosphate deposition. (c) Visualization by immunofluorescence analysis through confocal microscopy of a single differentiated cell (60x). (d) Image from three-dimensional stack analysis by confocal microscopy of blood-derived stem cells after ten days of osteogenic differentiation (in green, osteocalcin; in blue, DAPI: merged; (a), (b) $100 \mu$ m, (c) $\operatorname{bar}=8 \mu \mathrm{m}$ ).

TEM analysis also showed that osteoinduced BDSCs had a bulging cell body abundant in cytoplasm showing low N/P ratio. The cells extended many flat lamellipodia and dendritic filopodia with structural surface modifications, presenting many microvilli (Figures 2(c) and 2(d)).

The cytoplasm was rich in ribosomes and membranous organelles such as a well-developed ER and Golgi apparatus and many mitochondria exhibiting the orthodox configuration, with the inner compartment filled with abundant electron-dense matrix. Furthermore, EDX microanalysis allowed us to demonstrate the presence of calcium phosphate-containing vesicles and calciumcontaining granules within mitochondria. These "matrix vesicles" have been implicated in the mineralization of cartilage, bone, and dentin [14, 15, 36]. The observation that calcium phosphate and/or calcium is present both within mitochondria and intracellular vesicles suggests that a mechanism may exist in osteogenic cells by which ionic calcium (and perhaps phosphate) are transferred from mitochondria to intracellular vesicles, possibly via a simple process such as diffusion. This mechanism may play a role in bone apatite formation [37]. 


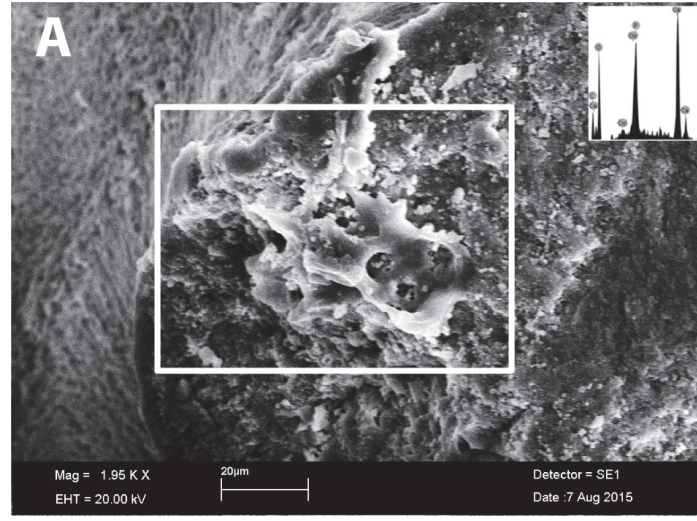

(a)

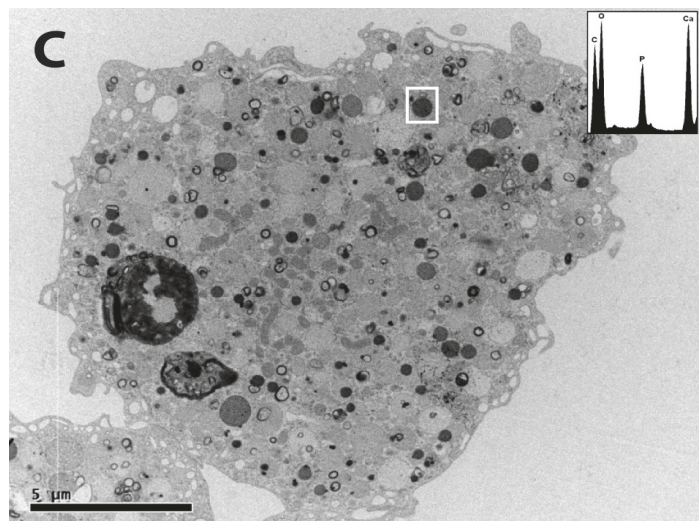

(c)

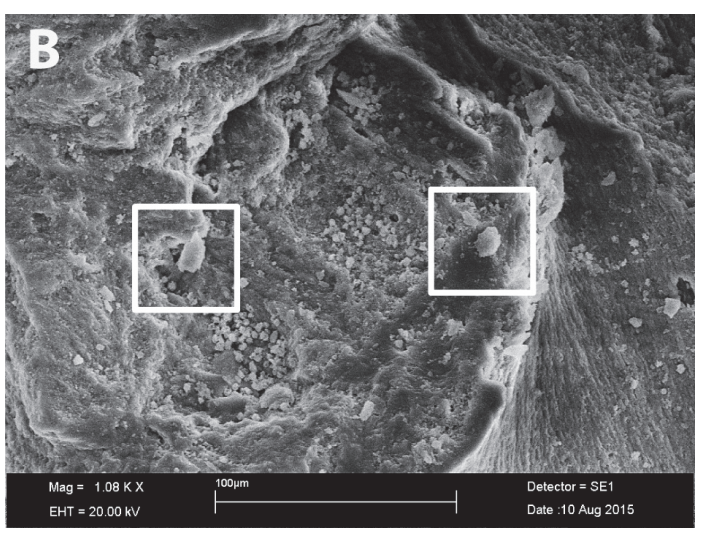

(b)

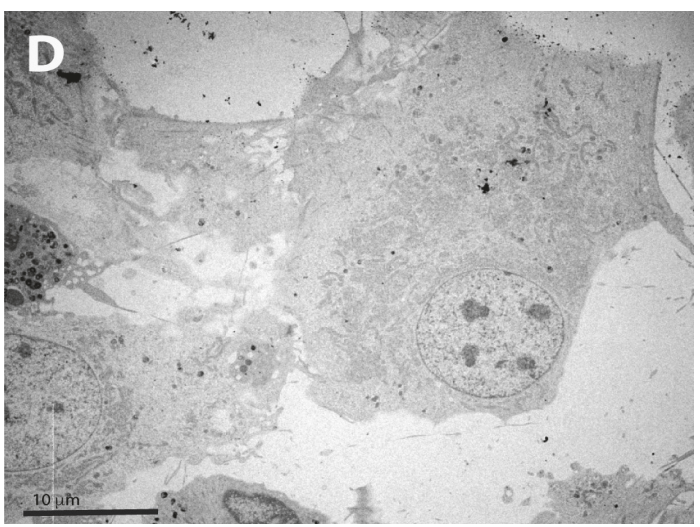

(d)

FIGURE 2: Electron microscopy demonstrating scaffold colonization of BDSCs differentiated with rapamycin, on bone scaffold. (a) Higher magnification of area indicated in the rectangle displays cells forming tissue-like agglomerates. Notably, electron micrograph captures an osteoblast matrix vesicle just as it initiated the exocytosis of hydroxyapatite (EDX spectrum). (b) Rectangles indicate differentiated osteoblast-like cells as shown by transmission electron microscopy analysis. (c) Higher magnification shows electron-dense granules within the mitochondria and the presence of calcium and phosphorus calcium phosphate aggregates typical of an osteoblast (EDX spectrum). (d) TEM image shows a typical osteoblast cell with flat lamellipodia and dendritic filopodia (bar $=20 \mu \mathrm{m}$ in (a), $100 \mu \mathrm{m}$ in (b), bar $=5 \mu \mathrm{m}$ in (c), and $10 \mu \mathrm{m}$ in (d)).

The aim of this new experimental protocol was to use a single molecule as an inducer and provide a simpler model to investigate the mechanisms underlying the osteogenesis of stem cells. The addition of bone scaffolds represented an improvement, resulting in a more uniform osteogenesis and exploring the ability of our cells to attach to and build on a bone matrix structure. These same bone scaffolds are routinely used in orthodontic procedures for numerous applications [38].

\subsection{Rapamycin Protocol Also Effective on Titanium Scaffold.} Once the ability of BDSCs to undergo osteogenic differentiation was confirmed, and a simplified differentiation protocol using rapamycin and bone matrix was established, a further step in our investigation was done in order to apply our protocol to a completely different material for osteogenic differentiation, namely, titanium.

Two titanium scaffolds were taken into consideration for these further experiments: the first being slices sampled from a titanium cylinder (type 1) and the second being pieces of a perforated sheet (type 2) both already commercially available as implants for orthodontic application in humans. After repeating our analysis with Alizarin Red S staining and immunocytochemical analysis for detecting osteocalcin, we found that cells successfully adhered to both. However, type 2 was selected as the more ideal scaffold as it allowed for better imaging, since the surface of the type 1 titanium slices had proven to be more irregular than expected and their display by confocal microscopy showed unsharp images due to cells being located on different planes (Figures 3 and 4). Time course experiments for osteocalcin expression were then performed to better evaluate the progression of colonization during the differentiation process.

To estimate the area of scaffold material coated by the cells, we set up a double analysis, combining densitometry with overlapping fluorescence signals as measured by two software that allowed to calculate the percentage of 


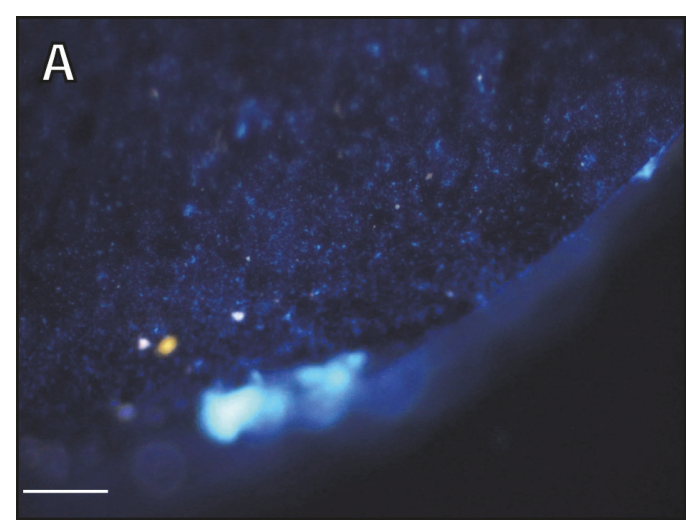

(a)

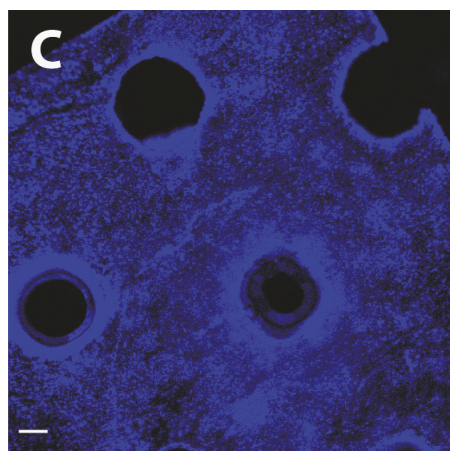

(c)

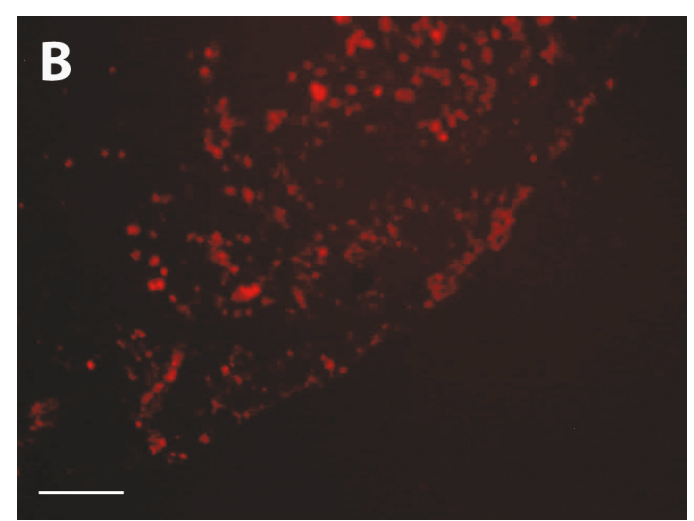

(b)

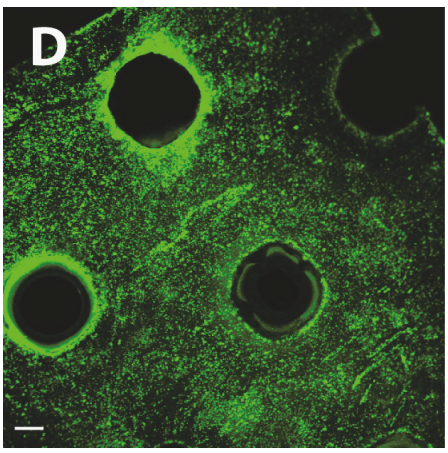

(d)

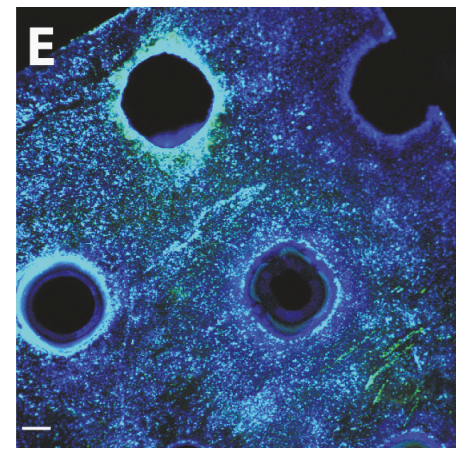

(e)

Figure 3: BDSCs osteogenic differentiation with rapamycin and titanium scaffolds. Nuclear and Alizarin Red S stainings on type 1 titanium after differentiation by confocal microscopy analysis ((a) and (b), resp.). Nuclear staining, osteocalcin expression, and merge analysis on type 2 titanium scaffold ((c), (d), and (e), resp.) (osteocalcin green, DAPI blue; (a), (b) bar=10 mm; (c), (d) $200 \mu \mathrm{m} 4 \mathrm{x})$.

area covered by the cells during their growth. This analysis was optimized by evaluating the intensity of image pixel brightness, removing the lowest light intensities due to round noise, and the highest due to artifacts (SD 3). Making a time-dependent analysis of the colonization process using two different confocal microscopy software allowed us to compare the percentage of metal surface covered by cells with the cell proliferation rate (Figure 5). The results show that not only BDSCs were able to colonize up to $90 \%$ of the titanium scaffold during their osteogenic reprogramming but also that the maximum peak of differentiation is obtained already during the first week. Furthermore, this simple imaging protocol allowed us to follow the differentiation process over time. The results obtained by overlaying the two signals in our experiments confirm the presence of the nuclei (in blue) and highlight the secreted osteocalcin (in green), enabling us to pinpoint how peak osteocalcin expression is reached as early as five days postinduction (Figure 6). In both cases, the differentiation timeframe of our experiments is significantly shorter than all those hitherto reported in similar works [39].

It is well known that the surface properties of a scaffold material can regulate stem cell fate [40-43] and, for example, induce osteoblast differentiation $[44,45]$. Theoretically, an ideal titanium implant would favor stem cell differentiation into mature osteoblasts for direct bone apposition. However, the development of such an ideal implant would require a more extensive investigation of the processes of attachment, colonization, and differentiation of these stem cells. We believe that our model may provide a first step in that direction.

\section{Conclusion}

With this work, we demonstrated that BDSCs are able to differentiate toward the osteogenic lineage not only by using a standard commercial kit but also by using rapamycin as single inducer molecule, both by itself and in the presence of bone or titanium scaffolds. We have shown that these cells are able to attach, proliferate, and differentiate on a nonpremodified, smooth titanium plate and that this scaffold does not interfere with the differentiation process. All of the materials used in these new protocols have been selected with an eye towards applicability in human therapy. This simple procedure, paired with its rapid differentiation time, might prove to be a valid model to study 


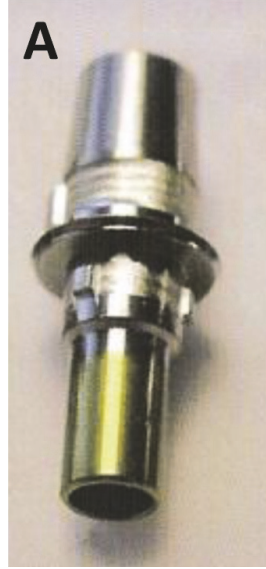

(a)

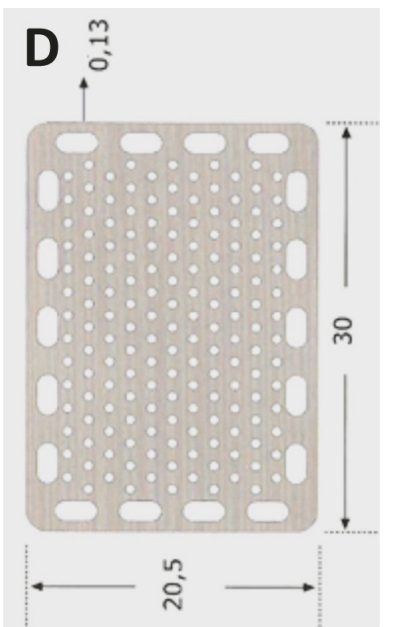

(d)

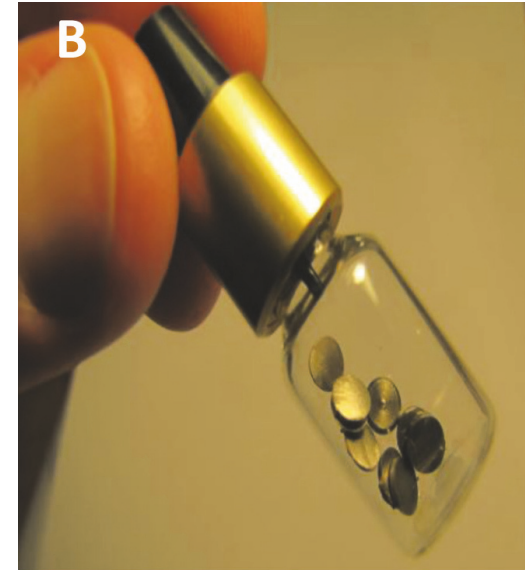

(b)

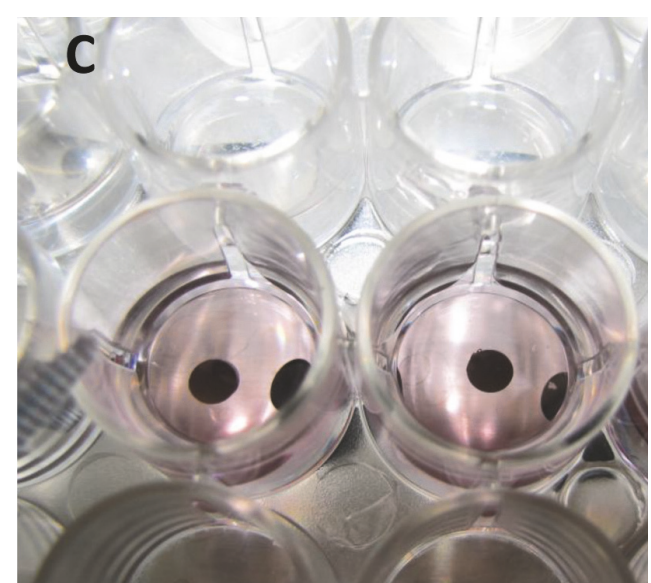

(c)

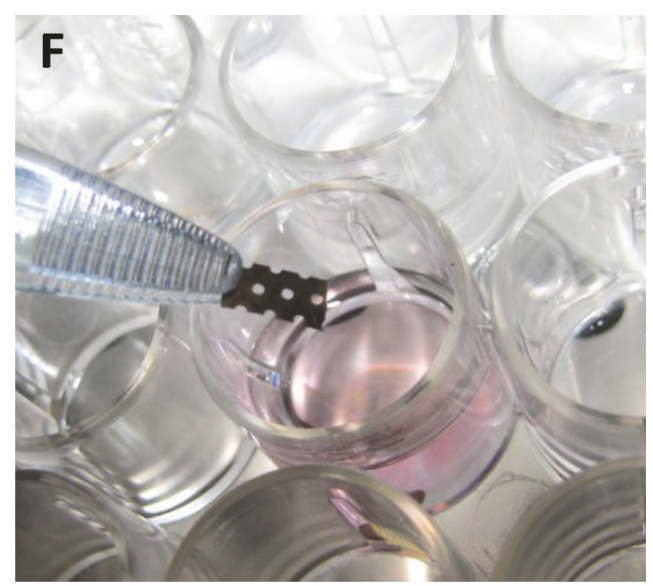

(f)

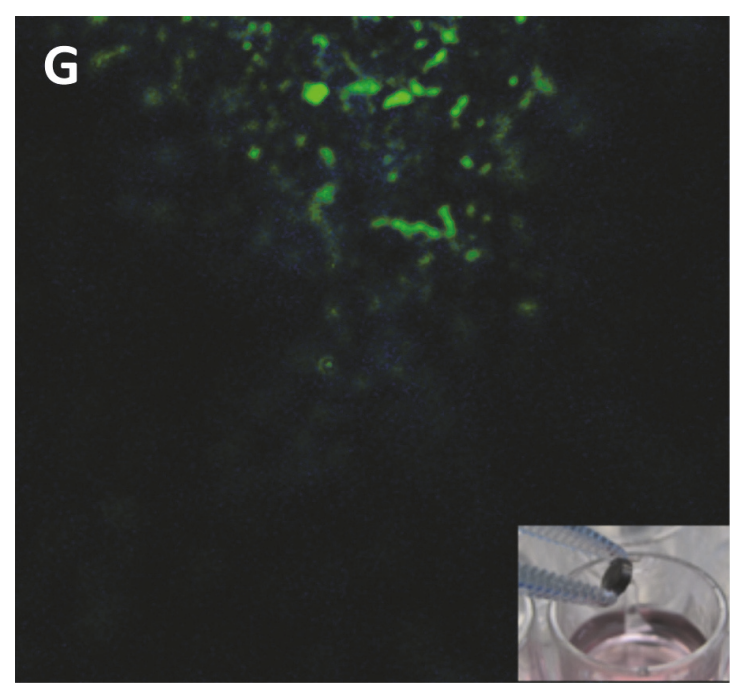

(g)

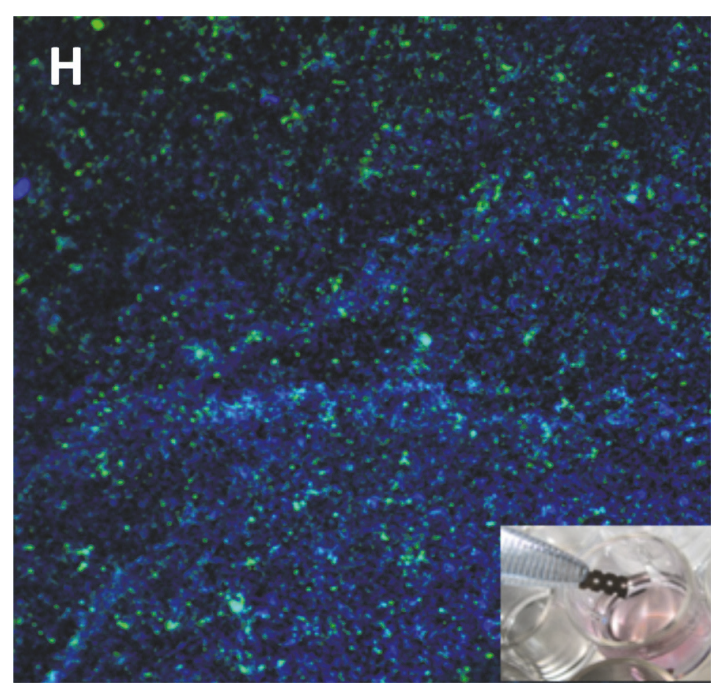

(h)

Figure 4: Titanium scaffolds: Titanium type 1. (a) The original endosseous implants before cutting, (b) titanium after cutting and sterilization, (c) titanium type 1 in a 24 -well plate before osteogenic differentiation experiments. Titanium type 2. (d) The original titanium grill before cutting. (e) The dimensions of the different perforations. (f) Titanium type 2 in a 24 -well plate before osteogenic differentiation experiments. (g) Titanium type 1 immunofluorescence images by confocal microscopy, the low resolution of the osteocalcin and nuclei signals are evident (green and blue, resp.). (h) Titanium type 2 immunofluorescence images by confocal microscopy, it is evident how the more flat surface of this scaffold is better suited for confocal microscopy analysis. 


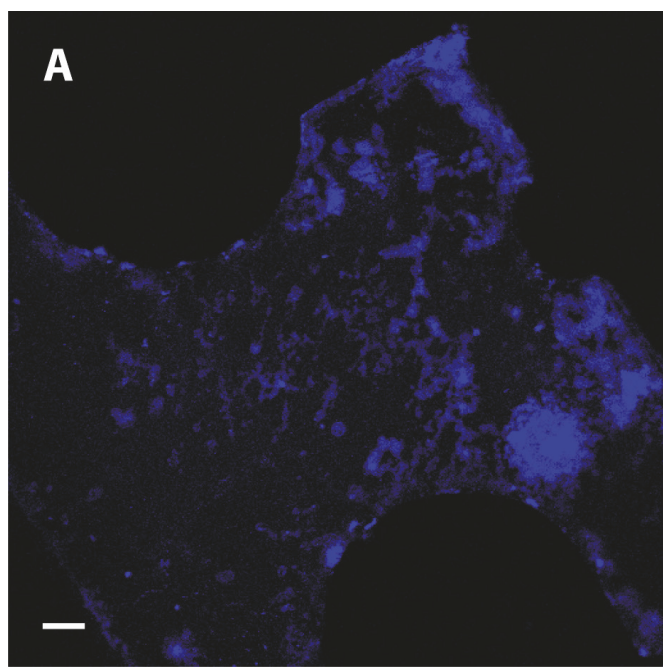

(a)

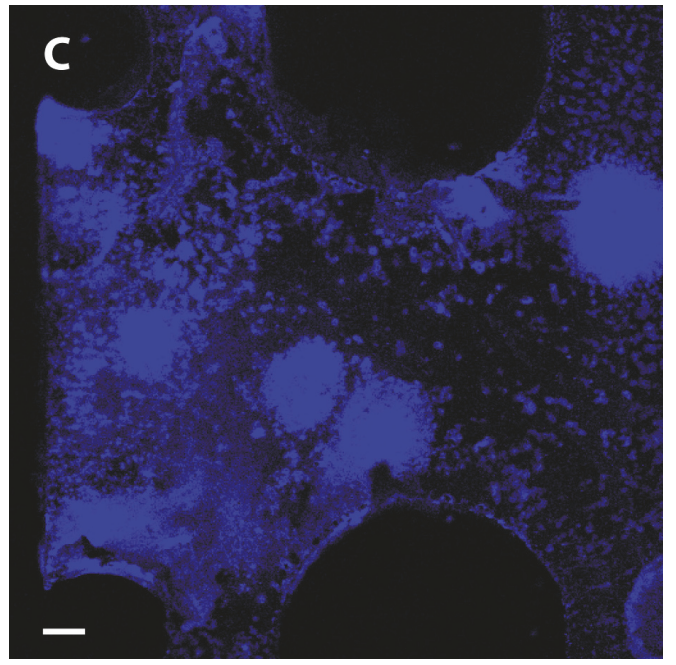

(c)

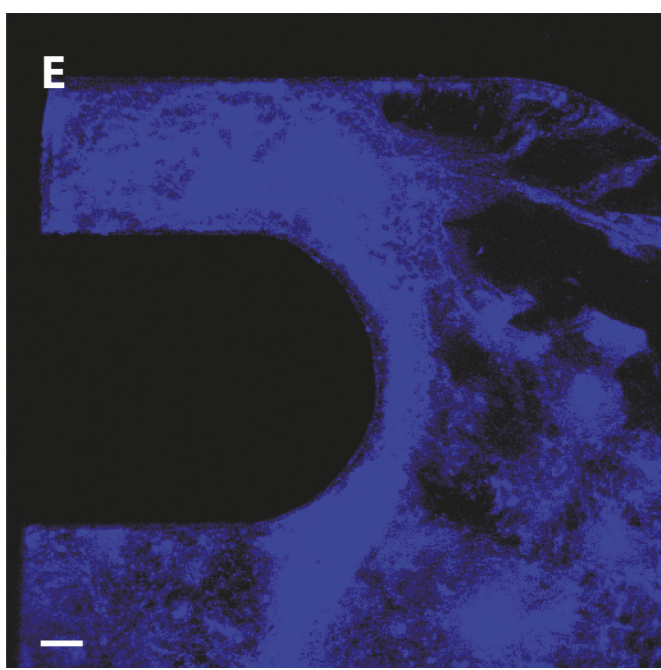

(e)

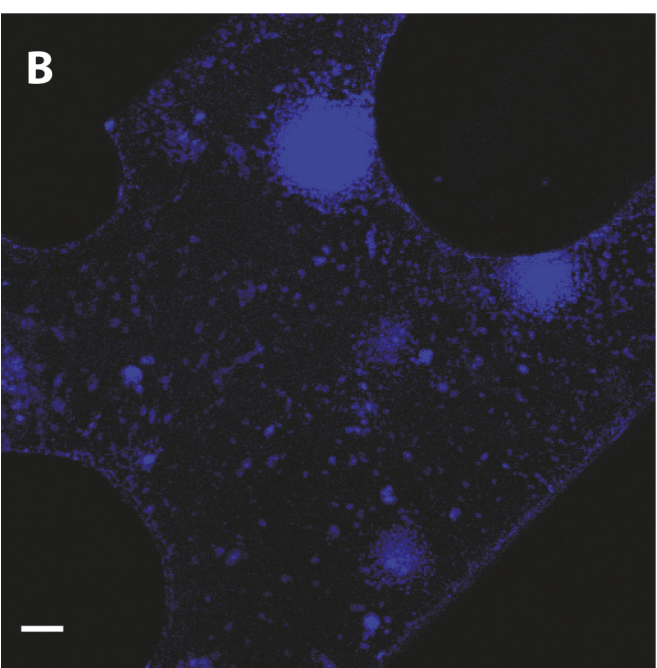

(b)

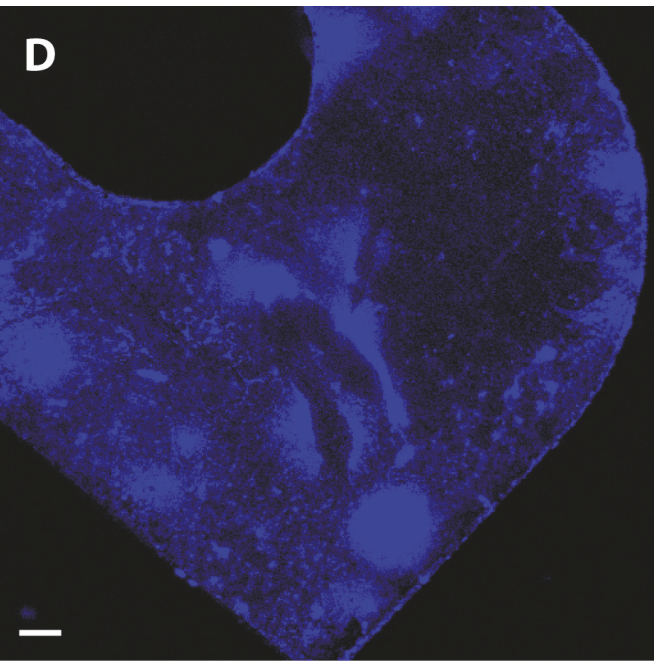

(d)

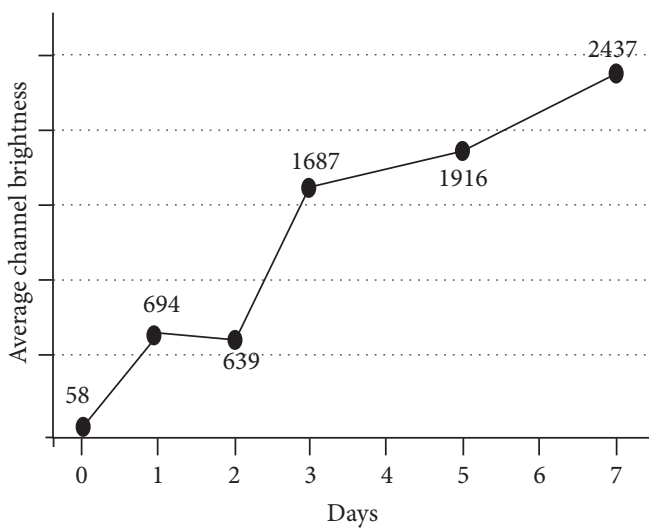

(f)

FIgURE 5: Time course experiments to detect the BDSCs capacity to adhere to the titanium scaffold. The increasing blue signal (DAPI) along time course analysis shows the cells' adhesion and growth on titanium ( (a) = after 1 day, $(\mathrm{b})=2$ days, (c) $=3$ days, $(\mathrm{d})=5$ days, (e) $=7$ days). (f) shows a graphical representation of (a)-(e) time course analysis performed by Nikon EZ-C1 viewer software. 

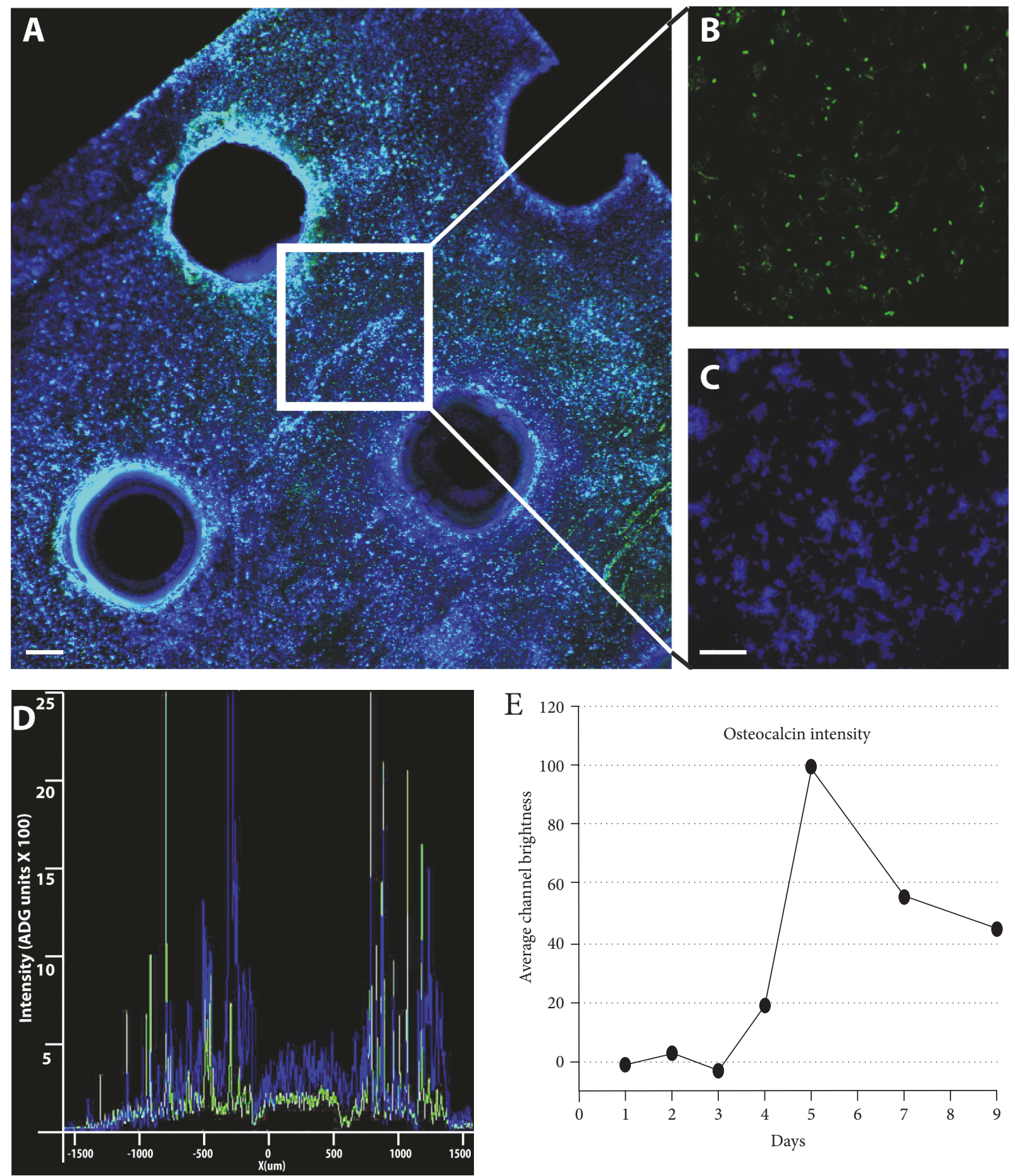

Figure 6: Analysis to detect BDSC capacity to differentiate on titanium scaffolds. (A) Merge analysis on type 2 titanium scaffold by osteocalcin expression ((B) osteocalcin green) and nuclear staining ((C) DAPI blue) to demonstrate BDSCs osteogenic differentiation. (D) shows a graphical representation of the two channel light intensity signals (expressed in ADG units $\times 100$ ). It is evident how the blue peaks (nuclear signal) overlap the green peaks (osteocalcin signal). (E) shows time course experiments for osteocalcin expression (expressed as average channel brightness) ((A): bar $=200 \mu \mathrm{m},(B),(C):$ bar $=50 \mu \mathrm{m})$.

the molecular mechanisms of osteogenesis while tailoring tissue engineering solutions to these new insights. BDSCs could prove to be a valid addition to the regenerative medicine toolbox, with the ultimate goal of creating new treatments to stimulate bone regeneration and improve prosthetic implantation. 


\section{Conflicts of Interest}

The authors declare that they have no conflicts of interest.

\section{Authors' Contributions}

Carpentieri Arianna, Cozzoli Eliana, and Acri Flavio have contributed equally to this work.

\section{Acknowledgments}

This work has been supported by the Italian Ministry of Research Grant no. “2007KAWXCL_002.” The authors also acknowledge the sources of private funding that have provided the basis for this study: "Progetto cellule staminali" NAST Centre.

\section{References}

[1] S. Ehnert, M. Glanemann, A. Schmitt et al., "The possible use of stem cells in regenerative medicine: dream or reality?," Langenbeck's Archives of Surgery, vol. 394, no. 6, pp. 985997, 2009.

[2] H. H. Maniar, A. A. Tawari, M. Suk, and D. S. Horwitz, "The current role of stem cells in orthopaedic surgery," Malaysian Orthopaedic Journal, vol. 9, no. 3, 2015.

[3] M. Tatullo, M. Marrelli, and F. Paduano, "Regenerative medicine in oral and maxillofacial surgery: the most important innovations in the clinical application of mesenchymal stem cell," International Journal of Medical Sciences, vol. 12, no. 1, pp. 72-77, 2015.

[4] A. Kokkonen, M. Ikävalko, R. Tiihonen, H. Kautiainen, and E. A. Belt, "High rate of osteolytic lesions in medium-term followup after the AES total ankle replacement," Foot and Ankle International, vol. 32, no. 2, pp. 168-175, 2011.

[5] P. Pelissier, P. Boireau, D. Martin, and J. Baudet, "Bone reconstruction of the lower extremity: complications and outcomes," Plastic and Reconstructive Surgery, vol. 111, no. 7, pp. 2223-2229, 2003.

[6] A. Schmitt, M. van Griensven, A. B. Imhoff, and S. Buchmann, "Application of stem cells in orthopedics," Stem Cells International, vol. 2012, Article ID 394962, 11 pages, 2012.

[7] E. Y. Waese, R. A. Kandel, and W. L. Stanford, "Application of stem cells in bone repair," Skeletal Radiology, vol. 37, no. 7, pp. 601-608, 2008.

[8] H. J. Jin, Y. K. Bae, M. Kim et al., "Comparative analysis of human mesenchymal stem cells from bone marrow, adipose tissue, and umbilical cord blood as sources of cell therapy," International Journal of Molecular Sciences, vol. 14, no. 9, pp. 17986-18001, 2013.

[9] G. Chamberlain, J. Fox, B. Ashton, and J. Middleton, "Concise review: mesenchymal stem cells: their phenotype, differentiation capacity, immunological features, and potential for homing," Stem Cells, vol. 25, no. 11, pp. 27392749, 2007.

[10] D. Trivanovic, A. Jaukovic, B. Popovic et al., "Mesenchymal stem cells of different origin: comparative evaluation of proliferative capacity, telomere length and pluripotency marker expression," Life Sciences, vol. 141, pp. 61-73, 2015.
[11] M. Al-Nbaheen, R. Vishnubalaji, D. Ali et al., "Human stromal (mesenchymal) stem cells from bone marrow, adipose tissue and skin exhibit differences in molecular phenotype and differentiation potential," Stem Cell Reviews, vol. 9, pp. 32-43, 2013.

[12] M. M. Bonab, K. Alimoghaddam, F. Talebian, S. H. Ghaffari, A. Ghavamzadeh, and B. Nikbin, "Aging of mesenchymal stem cell in vitro," BMC Cell Biology, vol. 7, p. 14, 2006.

[13] J. J. Bara, R. G. Richards, M. Alini, and M. J. Stoddart, "Bone marrow-derived mesenchymal stem cells change phenotype following in vitro culture: implications for basic research and the clinic," Stem Cells, vol. 32, pp. 1713-1723, 2014.

[14] P. V. Giannoudis, H. Dinopoulos, and E. Tsiridis, "Bone substitutes: an update," Injury, vol. 36, Supplement 3, pp. S20-S27, 2005.

[15] K. Arvidson, B. M. Abdallah, L. A. Applegate et al., "Bone regeneration and stem cells," Journal of Cellular and Molecular Medicine, vol. 15, no. 4, pp. 718-746, 2011.

[16] A. R. Amini, C. T. Laurencin, and S. P. Nukavarapu, "Bone tissue engineering: recent advances and challenges," Critical Reviews in Biomedical Engineering, vol. 40, no. 5, pp. 363408, 2012.

[17] X. Yu, X. Tang, S. V. Gohil, and C. T. Laurencin, "Biomaterials for bone regenerative engineering," Advanced Healthcare Materials, vol. 4, no. 9, pp. 1268-1285, 2015.

[18] K. W. Lee, J. Y. Yook, M. Y. Son et al., "Rapamycin promotes the osteoblastic differentiation of human embryonic stem cells by blocking the mTOR pathway and stimulating the BMP/ Smad pathway," Stem Cells and Development, vol. 19, no. 4, pp. 557-568, 2010

[19] T. Ogawa, M. Tokuda, K. Tomizawa et al., "Osteoblastic differentiation is enhanced by rapamycin in rat osteoblast like osteosarcoma (ROS 17/2.8) cells," Biochemical and Biophysical Research Communications, vol. 249, pp. 226-230, 1998.

[20] A. Pantovic, A. Krstic, K. Janjetovic et al., "Coordinated timedependent modulation of AMPK/Akt/mTOR signaling and autophagy controls osteogenic differentiation of human mesenchymal stem cells," Bone, vol. 52, no. 1, pp. 524-531, 2013.

[21] M. Fakhry, E. Hamade, B. Badran, R. Buchet, and D. Magne, "Molecular mechanisms of mesenchymal stem cell differentiation towards osteoblasts," World Journal of Stem Cells, vol. 5, no. 4, pp. 136-148, 2013.

[22] E. J. Carbone, K. Rajpura, T. Jiang, C. T. Laurencin, and K. W. Lo, "Regulation of bone regeneration with approved small molecule compounds," Advances in Regenerative Biology, vol. 1, article 25276, 2014.

[23] G. Alaimo, E. Cozzoli, G. Marfe et al., "Blood-derived stem cells (BDSCs) plasticity: in vitro hepatic differentiation," Journal of Cellular Physiology, vol. 228, no. 6, pp. 1249-1254, 2013.

[24] G. Marfe, M. Massaro-Giordano, M. Ranalli et al., "Blood derived stem cells: an ameliorative therapy in veterinary ophthalmology," Journal of Cellular Physiology, vol. 227, pp. 1250-1256, 2012.

[25] G. Marfe, G. Rotta, L. De Martino et al., "A new clinical approach: use of blood-derived stem cells (BDSCs) for superficial digital flexor tendon injuries in horses," Life Sciences, vol. 90, pp. 825-830, 2012.

[26] J. H. Spaas, A. Gambacurta, M. Polettini et al., "Purification and expansion of stem cells from equine peripheral blood, with clinical applications," Flemish Veterinary Journal, vol. 80, pp. 129-136, 2011. 
[27] N. Jaiswal, S. E. Haynesworth, A. I. Caplan, and S. P. Bruder, "Osteogenic differentiation of purified, culture-expanded human mesenchymal stem cells in vitro," Journal of Cellular Biochemistry, vol. 64, pp. 295-312, 1997.

[28] P. A. Zuk, M. Zhu, P. Ashjian et al., "Human adipose tissue is a source of multipotent stem cells," Molecular Biology of the Cell, vol. 13, no. 12, pp. 4279-4295, 2002.

[29] A. Carpentieri, E. Cozzoli, M. Scimeca, E. Bonanno, A. M. Sardanelli, and A. Gambacurta, "Differentiation of human neuroblastoma cells toward the osteogenic lineage by mTOR inhibitor," Cell Death and Disease, vol. 6, 2015.

[30] S. Di Silvestro, F. D. Isep, N. Ferrigno, S. Fortini, and C. Acri, "Sterilizzazione degli impianti "Valutazione della variazione dell'energia di superficie di impianti in titanio in relazione all'utilizzazione delle microonde"," pp. 1-30, 2004, Guidelines ISTISAN 04/2.

[31] C. G. Jones, "Scanning electron microscopy: preparation and imaging for SEM," Methods in Molecular Biology, vol. 915, pp. 1-20, 2012.

[32] M. Scimeca, A. Orlandi, I. Terrenato, S. Bischetti, and E. Bonanno, "Assessment of metal contaminants in non-small cell lung cancer by EDX microanalysis," European Journal of Histochemistry, vol. 58, no. 3, p. 2403, 2014.

[33] E. S. Reynolds, "The use of lead citrate at high $\mathrm{pH}$ as an electron opaque stain based on metal chelation," The Journal of Cell Biology, vol. 17, pp. 208-212, 1963.

[34] M. Scimeca, A. Pietroiusti, F. Milano et al., "Elemental analysis of histological specimens: a method to unmask nano asbestos fibers," European Journal of Histochemistry, vol. 60, no. 1, p. 2573, 2016.

[35] F. Langenbach and J. Handschel, "Effects of dexamethasone, ascorbic acid and $\beta$-glycerophosphate on the osteogenic differentiation of stem cells in vitro," Stem Cell Research \& Therapy, vol. 4, no. 5, p. 117, 2013.

[36] E. Davies, "Bone bonding at natural and biomaterial surfaces," Biomaterials, vol. 28, no. 34, pp. 5058-5067, 2008.

[37] S. Boonrungsiman, E. Gentleman, R. Carzaniga et al., "The role of intracellular calcium phosphate in osteoblast-mediated bone apatite formation," Proceedings of the National Academy of Sciences of the United States of America, vol. 109, no. 35, pp. 14170-14175, 2012.

[38] E. A. Abou Neel, W. Chrzanowski, V. M. Salih, H. W. Kim, and J. C. Knowles, "Tissue engineering in dentistry," Journal of Dentistry, vol. 42, no. 8, pp. 915-928, 2014.

[39] E. Castrén, T. Sillat, S. Oja et al., "Osteogenic differentiation of mesenchymal stromal cells in two-dimensional and three-dimensional cultures without animal serum," Stem Cell Research \& Therapy, vol. 6, no. 1, p. 167, 2015.

[40] J. Kim and A. Atala, "Scaffold technologies for controlling cell behavior in tissue engineering," Biomedical Materials, vol. 8, no. 1, article 010201, 2013.

[41] M. L. Wang, R. Tuli, P. A. Manner, P. F. Sharkey, D. J. Hall, and R. S. Tuan, "Direct and indirect induction of apoptosis in human mesenchymal stem cells in response to titanium particles," Journal of Orthopaedic Research, vol. 21, no. 4, pp. 697-707, 2013.

[42] M. Wang, T. Chen, S. Lu et al., "Osteogenic comparison on selective laser melting printed and sandblasting-acid-etching Ti substrates for customized implant applications," Science of Advanced Materials, vol. 9, no. 5, pp. 705-714, 2017.
[43] P. Caroppi, F. Sinibaldi, E. Santoni et al., "The 40s omega-loop plays a critical role in the stability and the alkaline conformational transition of cytochrome c," Journal of Biological Inorganic Chemistry, vol. 9, pp. 997-1006, 2004.

[44] L. Xia, K. Lin, X. Jiang et al., "Effect of nano-structured bioceramic surface on osteogenic differentiation of adipose derived stem cells," Biomaterials, vol. 35, no. 30, pp. $8514-$ 8527, 2014.

[45] M. Wang, X. Cheng, W. Zhu, B. Holmes, M. Keidar, and L. G. Zhang, "Design of biomimetic and bioactive cold plasma-modified nanostructured scaffolds for enhanced osteogenic differentiation of bone marrow-derived mesenchymal stem cells," Tissue Engineering Part a, vol. 20, no. 5-6, pp. 1060-1071, 2014. 

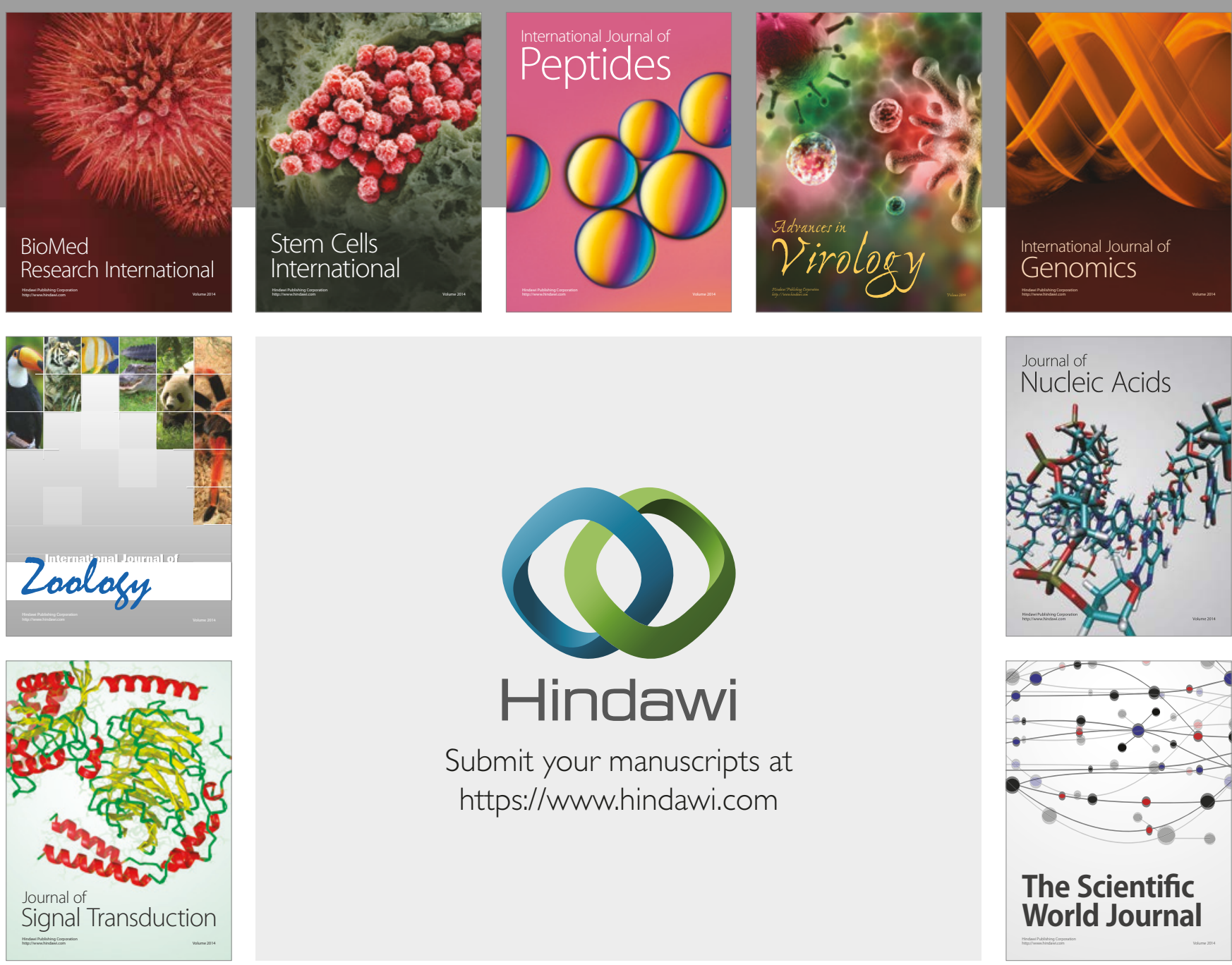

Submit your manuscripts at

https://www.hindawi.com
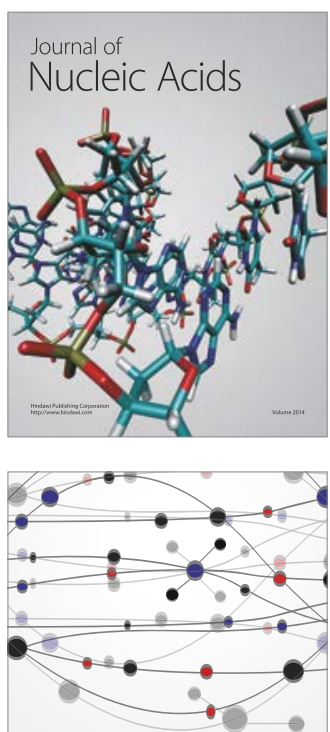

The Scientific World Journal

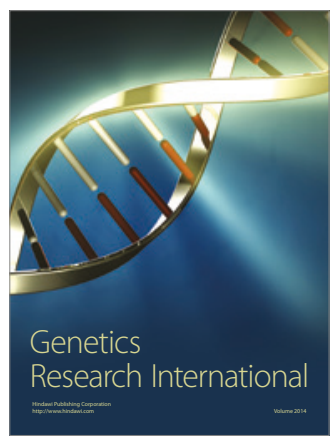

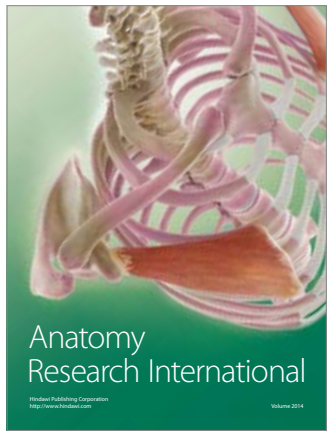

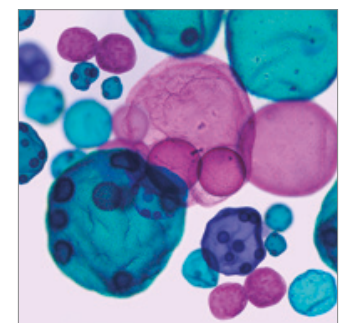

International Journal of Microbiology
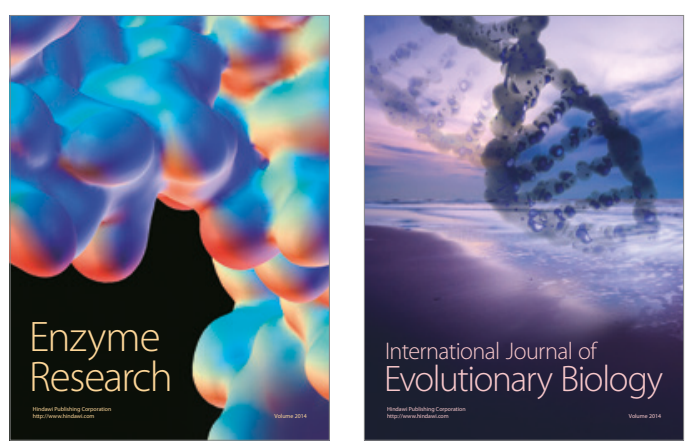
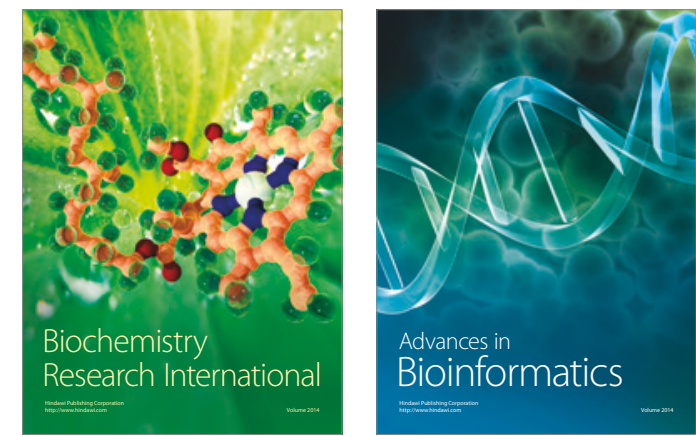

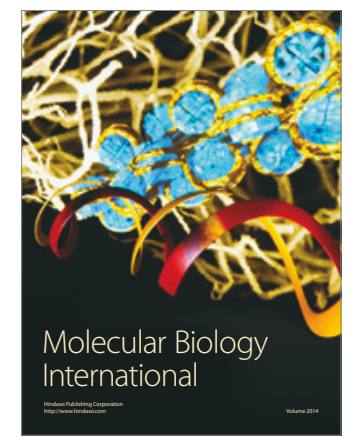

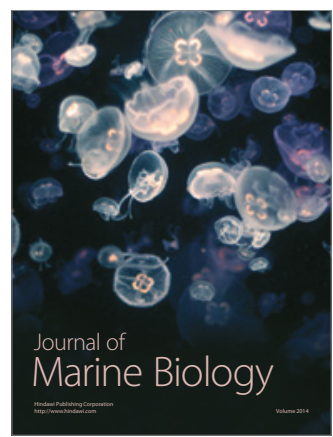

